\title{
A New-Trend Model-Based to Solve the Peak Power Problems in OFDM Systems
}

\author{
Ashraf A. Eltholth, ${ }^{1}$ Adel R. Mekhail, ${ }^{1}$ A. Elshirbini, ${ }^{1}$ M. I. Dessouki, ${ }^{2}$ and A. I. Abdelfattah ${ }^{3}$ \\ ${ }^{1}$ National Telecommunication Institute, P.O. Box 7070, 11768, Cairo, Egypt \\ ${ }^{2}$ Department of Communication Engineering, Faculty of Electronic Engineering, Minufiya University, Minufiya, Egypt \\ ${ }^{3}$ Department of Electrical Communication, Faculty of Engineering, Mansoura University, Mansoura, Egypt
}

Correspondence should be addressed to Ashraf A. Eltholth, eau_25@yahoo.com

Received 29 August 2007; Revised 30 April 2008; Accepted 6 July 2008

Recommended by Adel Alimi

\begin{abstract}
The high peak to average power ration (PAR) levels of orthogonal frequency division multiplexing (OFDM) signals attracts the attention of many researchers during the past decade. Existing approaches that attack this PAR issue are abundant, but no systematic framework or comparison between them exist to date. They sometimes even differ in the problem definition itself and consequently in the basic approach to follow. In this paper, we propose a new trend in mitigating the peak power problem in OFDM system based on modeling the effects of clipping and amplifier nonlinearities in an OFDM system. We showed that the distortion due to these effects is highly related to the dynamic range itself rather than the clipping level or the saturation level of the nonlinear amplifier, and thus we propose two criteria to reduce the dynamic range of the OFDM, namely, the use of MSK modulation and the use of Hadamard transform. Computer simulations of the OFDM system using Matlab are completely matched with the deduced model in terms of OFDM signal quality metrics such as BER, ACPR, and EVM. Also simulation results show that even the reduction of PAR using the two proposed criteria is not significat, the reduction in the amount of distortion due to HPA is truley delightful.
\end{abstract}

Copyright (C) 2008 Ashraf A. Eltholth et al. This is an open access article distributed under the Creative Commons Attribution License, which permits unrestricted use, distribution, and reproduction in any medium, provided the original work is properly cited.

\section{Introduction}

In OFDM systems, the combination of different signals with different phase and frequency gives a large dynamic range that is used to be characterized by a high PAR, which results in severe clipping effects and nonlinear distortion if the composite time signal is amplified by a power amplifier, which have nonlinear transfer function. This degrades the performance of an OFDM system. A measure of the degradation can be very helpful in evaluating the performance of a given system, and in designing a signaling set that avoids degradation. The high PAR sets strict requirements for the linearity of the PA. In order to limit the adjacent channel leakage, it is desirable for the PA to operate in its linear region. High linearity requirement for the PA leads to lowpower efficiency and therefore to high-power consumption. PAs are divided into classes according to the biasing used. A class (A) amplifier is defined as an amplifier that is biased so that the current drawn from the battery is equal to the maximum output current. The class (A) amplifier is the most linear of all amplifier types, but the maximum efficiency of the amplifier is limited to $50 \%$. In reality, due to the fact that the amplitude of the input signal is most of the time much less than its maximum value, the efficiency is much less than the theoretical maximum, that is, only a few percent. This poor efficiency causes high-power consumption, which leads to warming in physical devices. This is a problem especially in a base station where the transmitted power is usually high. To achieve a better efficiency, the amplifier can be biased so that current flows only half the time on either the positive or negative half cycle of the input signal. An amplifier biased like this is called a class (B) amplifier. The cost of the increased efficiency is worse linearity than in a class (A) amplifier. High demands on linearity make class (B) unsuitable for a system with high PAR. On the other hand, the large scale of the input signal makes it difficult to bias an amplifier operating in class (A). In practice, the amplifier is a compromise between classes (A) and (B), and is called a class (AB) amplifier [1]. 
Several options appear in the literature related with OFDM systems and nonlinearities. PAR reduction using clipping or coding or phase optimization techniques or a combination of any two of them [2], are the tools to combat nonlinearities used in the transmitter. Also a good work have been done in modeling the performance of OFDM systems with power amplifiers in [1], but it has assumed the OFDM signal to have Gaussian distribution which is not very accurate description of the composite time OFDM signal. In this paper, the effect of power amplifier nonlinearities is modeled in OFDM systems. We showed that the distortion due to these effects is highly related to the dynamic range itself rather than the clipping level or the saturation level of the nonlinear amplifier, and thus we propose two criteria to reduce the dynamic range of the OFDM, namely, the use of N-MSK modulation and the use of Hadamard transform. Computer simulations of the OFDM system using Matlab are completely matched with the deduced model in terms of OFDM signal quality metrics such as BER, ACPR, and EVM. Also simulation results show that even the reduction of PAR using the two proposed criteria is not significant, the reduction in the distortion due to HPA is significant. Section 2 depicts OFDM signal statistical properties, while Section 3 introduces power amplifier models. The derivation of clipping noise and distortion is presented in Section 4, OFDM signal quality metrics are discussed in Section 5. Simulation results are included in Section 5. Finally, the conclusions are drawn.

\section{OFDM Signal Statistical Properties}

It is well known that according to the central limit theory that the real and imaginary parts of the OFDM signal completely agree with the normal distribution and consequently its absolute agrees with the Rayleigh distribution with probability density function expressed by

$$
P(x)=\frac{x}{s} e^{-x^{2} / 2 s^{2}}, \quad x \in[0, \infty]
$$

where $s$ is a parameter, and mean $\mu=s \sqrt{\pi / 2}$, and variance $\sigma^{2}=((4-\pi) / 2) s^{2}$.

Figure 1 explicitly shows that the measured amplitude histogram of the inphase component/quadrature component for a 256 subcarrier OFDM signal.

While Figure 2 shows the histogram of the OFDM signal absolute.

It is clear that the distribution in Figure 1 obeys a Gaussian distribution, while that in Figure 2 obeys a Rayleigh distribution.

\section{Power Amplifier Model}

A short description of power amplifier models will be given in this section. Consider an input signal in polar coordinates as [1]

$$
x=\rho e^{j \varphi} .
$$

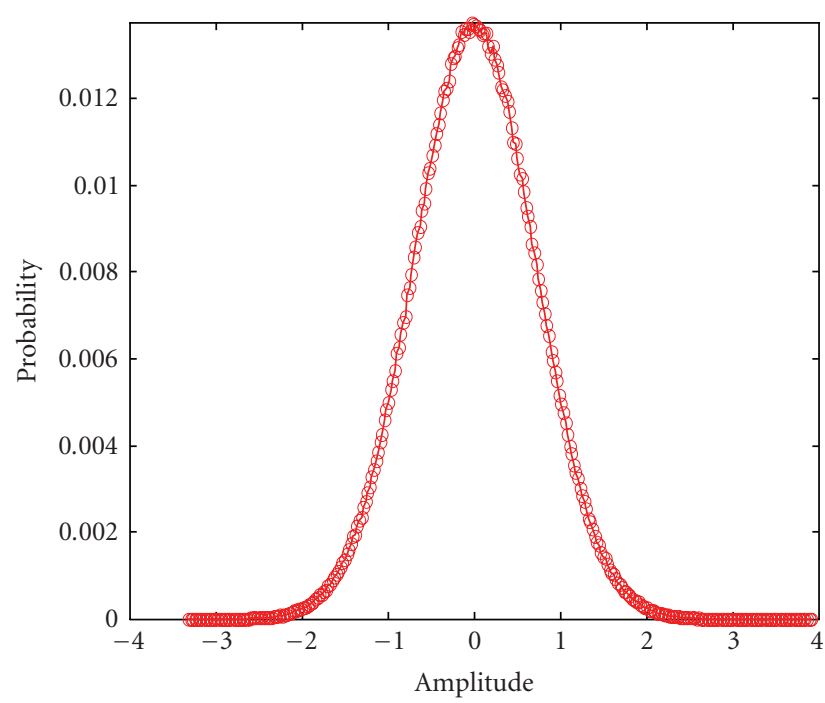

FIgURE 1: Inphase/quadrature component histogram of a 256 subcarrier OFDM signal.

$(\mathrm{dB})$

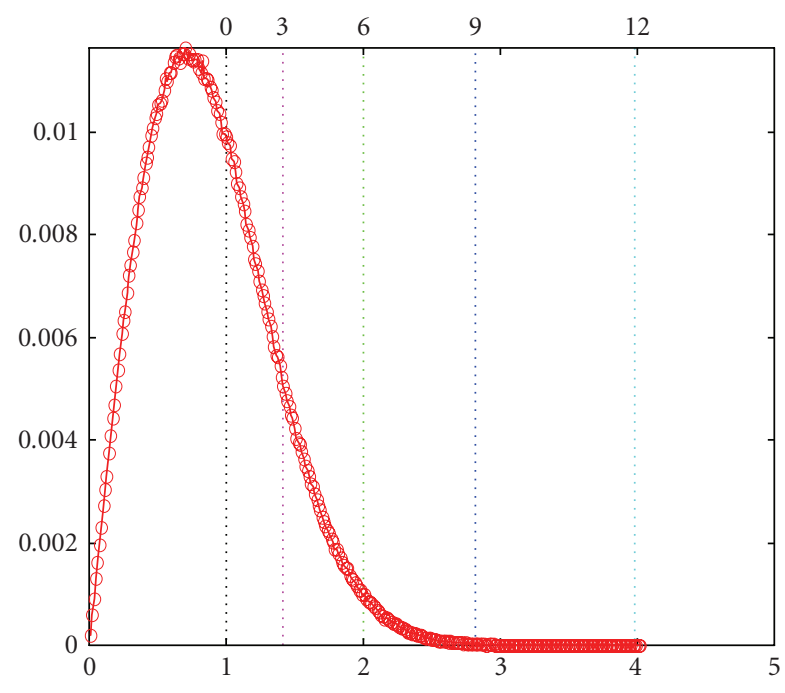

Figure 2: Amplitude histogram of a 256 subcarrier OFDM signal.

The output of the power amplifier can be written as

$$
g(x)=M(\rho) e^{j(\phi+P(\rho))},
$$

where $M(\rho)$ represents the AM/AM conversion and $P(\rho)$ the $\mathrm{AM} / \mathrm{PM}$ conversion characteristics of the power amplifier.

Several models have been developed for nonlinear power amplifiers, the most commonly used ones are as follows.

\subsection{Limiter Transfer Characteristics}

A Limiter (clipping) amplifier is expressed as [3]

$$
M(\rho)= \begin{cases}\rho, & |\rho|<A, \\ A, & |\rho| \geq A,\end{cases}
$$




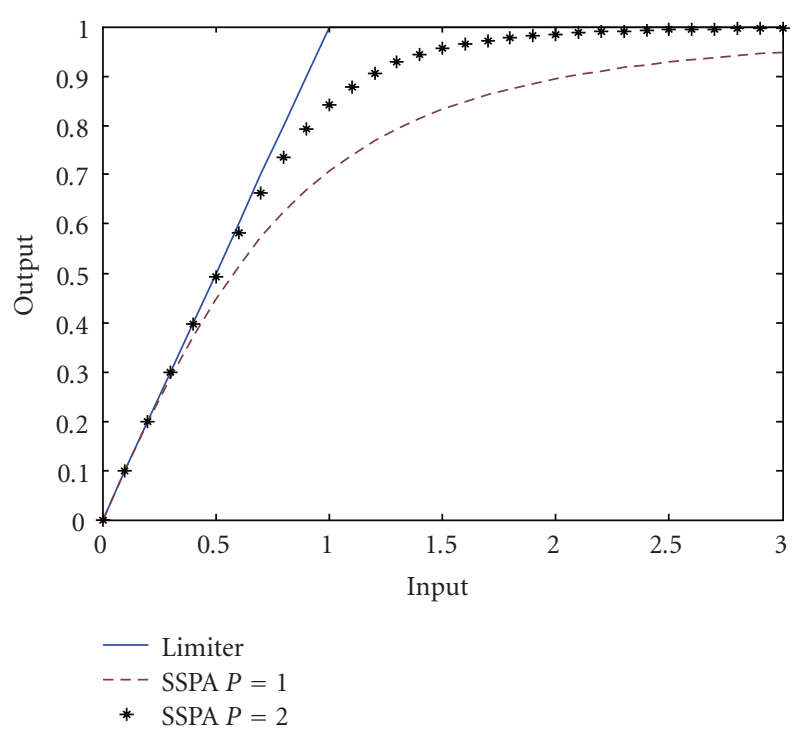

Figure 3: AM/AM conversion of HPA.

where $A$ is the clipping level. This model does not consider $\mathrm{AM} / \mathrm{PM}$ conversion.

\subsection{Solid-State Power Amplifier (SSPA)}

The conversion characteristics of solid-state power amplifier are modeled by Rapp's SSPA with characteristic [3]:

$$
v_{\text {out }}=\frac{v_{\text {in }}}{\left(1+\left(\left|v_{\text {in }}\right| / v_{\text {sat }}\right)^{2 p}\right)^{1 / 2 p}}
$$

where $v_{\text {out }}$ and $v_{\text {in }}$ are complex $i / p \& o / p, v_{\text {sat }}$ is the output at the saturation point $\left(v_{\text {sat }}=A / \sqrt{2}\right)$, and $P$ is "knee factor" that controls the smoothness of the transition from the linear region to the saturation region of characteristic curve (a typical value of $P$ is 1 ).

Figure 3 shows the AM/AM conversion of the two described models with $A=1$. It is clear from the figure; as the value of knee factor increases the SSPA model approaches the limiter model.

One problem with these methods is that the special model of nonlinear device requires expensive and time consuming experimental measurements to identify model parameters. On the contrary, simple measures of nonlinearity directly related to a low-order polynomial model, such as third- and fifth-order intersect points, are usually available to a system designer at the early stage of specification definition or link budget analysis. The third-order nonlinearity model can be described by the Taylor series as [4]

$$
y(t)=a_{o}+a_{1} x(t)+a_{2} x^{2}(t)+a_{3} x^{3}(t)
$$

where $x(t)$ is the input, $y(t)$ is the output signal, and $\left\{a_{n}\right\}$ are Taylor series coefficients.

The nonlinearity of radio frequency circuits is often expressed in terms of the third-order intercept point $\left(A_{\mathrm{IP} 3}\right)$.

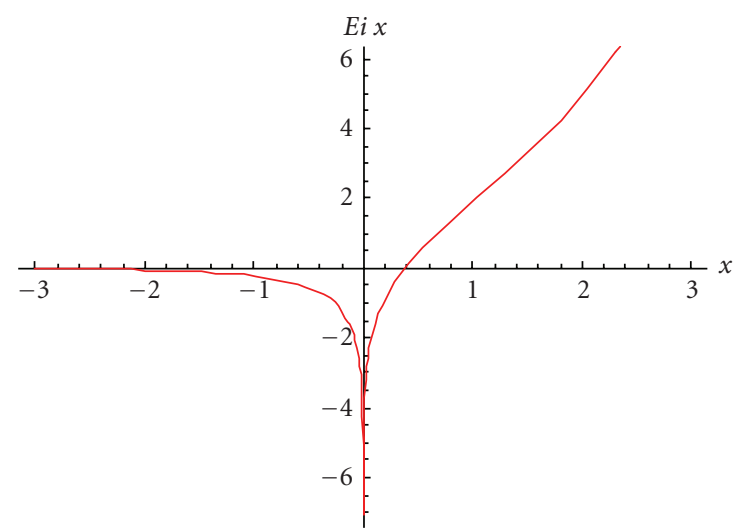

FIgURE 4: Exponential integral.

It can be shown that $A_{\mathrm{IP} 3}$ and parameters of third-order nonlinearity model are related as [4]

$$
A_{\mathrm{IP} 3}=\sqrt{4 a_{1} / 3\left|a_{3}\right|} \text {. }
$$

In this study, we only consider the third-order model of nonlinearity, since the third-order nonlinearity is usually dominated in real systems.

In this paper, we have simulated Rapp's SSPA model, and then deduced the third-order nonlinearity model to formulate the relation between $v_{\text {sat }}$ in Rapp's SSPA model and $A_{\mathrm{IP} 3}$ in $\mathrm{dB}$ widely used to express nonlinearity and we have got

$$
v_{\mathrm{sat}}=p_{1}\left(A_{\mathrm{IP} 3}\right)^{3}+p_{2}\left(A_{\mathrm{IP} 3}\right)^{2}+p_{3}\left(A_{\mathrm{IP} 3}\right)+p_{4},
$$

where

$$
\begin{gathered}
p_{1}=0.001936, \quad p_{2}=-0.09044, \\
p_{3}=1.438, \quad p_{4}=-5.197 .
\end{gathered}
$$

\section{Nonlinearity Distortion Analysis}

Here, we will analyze the effect of nonlinear amplifier on the OFDM signal, first: we will consider the NLA as a limiter that is expressed by

$$
g(x)= \begin{cases}x, & x \leq a \\ a, & x>a\end{cases}
$$

and thus the distortion due to the NLA as a limiter can be represented by an extra Gaussian noise with variance $\sigma_{\text {limiter }}^{2}$, 
where

$$
\begin{aligned}
\sigma_{\text {limiter }}= & \int_{a}^{\infty}(x-a)^{2} P(x) d x=\int_{a}^{\infty}(x-a)^{2} \frac{x}{s} e^{-x^{2} / 2 s^{2}} d x, \\
\therefore \sigma_{\text {limiter }}= & \frac{1}{s}\left(e^{-x^{2} / 2 s^{2}}\left(-x^{2} s^{2}-\left(a^{2}+2 s^{2}\right) s^{2}+2 a x s^{2}\right)\right. \\
& \left.\quad-a \sqrt{2 \pi} s^{3} \operatorname{erf}\left(\frac{x}{2 s}\right)\right)_{a}^{\infty} \\
= & \frac{1}{s}\left(0-a \sqrt{2 \pi} s^{3}\right) \\
& -\frac{1}{s}\left(e^{-a^{2} / 2 s^{2}}\left(-a^{2} s^{2}-\left(a^{2}+2 s^{2}\right) s^{2}+2 a^{2} s^{2}\right)\right. \\
& \left.\quad-a \sqrt{2 \pi} s^{3} \operatorname{erf}\left(\frac{a}{2 s}\right)\right) .
\end{aligned}
$$

This leads to

$$
\sigma_{\text {limiter }}=a \sqrt{2 \pi} s^{2}\left(\operatorname{erf}\left(\frac{a}{\sqrt{2} s}\right)-1\right)+2 s^{3} e^{-a^{2} / 2 s^{2}} .
$$

And finally

$$
\sigma_{\text {limiter }}=2 s^{3} e^{-a^{2} / 2 s^{2}}+a \sqrt{2 \pi} s^{2} \operatorname{erf} c\left(\frac{a}{\sqrt{2} s}\right) .
$$

If we consider the solid-state power amplifier model given by Rapp

$$
g(x)=\frac{x}{\left(1+\left(x / v_{\mathrm{sat}}\right)^{2 p}\right)^{1 / 2 p}},
$$

and let $p=1$ and $v_{\text {sat }}=a$, the NLA distortion can be represented by

$$
\begin{aligned}
\sigma_{\mathrm{SSPA}}=\int_{0}^{\infty}(x-g(x))^{2} P(x) d x \\
=\int_{0}^{\infty}\left(x-\frac{x}{\sqrt{1+\left(x / v_{\mathrm{sat}}\right)^{2}}}\right)^{2} \frac{x}{s} e^{-x^{2} / 2 s^{2}} d x, \\
\sigma_{\mathrm{SSPA}}=\frac{1}{2 s}\left(-2 a^{2} s^{2}+4 s^{4}+a^{4} e^{a^{2} / 2 s^{2}} E i\left[-\frac{a^{2}}{2 s^{2}}\right]\right. \\
\quad-2 \sqrt{a^{2}} e^{-a^{2} / 2 s^{2}} \sqrt{2 \pi} s\left(a^{2}-s^{2}\right) \operatorname{erf}\left[\frac{a}{\sqrt{2} s}\right] \\
\left.+2 \sqrt{a^{2}} e^{-a^{2} / 2 s^{2}} \sqrt{2 \pi} s\left(a^{2}-s^{2}\right)\right),
\end{aligned}
$$

where $\operatorname{Ei}(x)$ is the exponential integral, is defined as $E_{i}(x)=$ $\int_{-\infty}^{x}\left(e^{t} / t\right) d t$. It is plotted as in Figure 4.

$$
\begin{aligned}
\therefore \sigma_{\mathrm{SSPA}}=\frac{1}{2 s}( & 2 a e^{-a^{2} / 2 s^{2}} \sqrt{2 \pi} s\left(a^{2}-s^{2}\right)\left(1-\operatorname{erf}\left[\frac{a}{\sqrt{2} s}\right]\right) \\
& \left.+a^{4} e^{a^{2} / 2 s^{2}} E i\left[-\frac{a^{2}}{2 s^{2}}\right]-2 a^{2} s^{2}+4 s^{4}\right),
\end{aligned}
$$

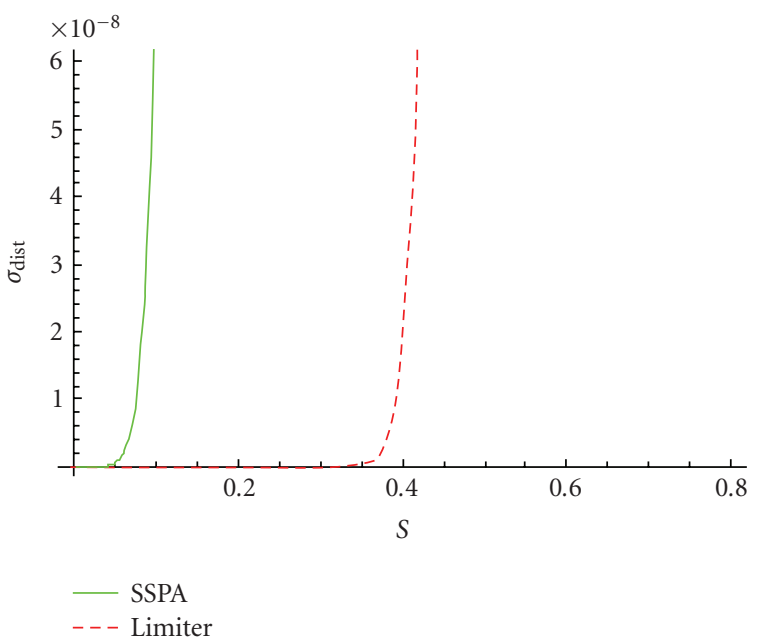

FIgURE 5: Nonlinear amplifier distortion versus $s$, with saturation level $a=2\left(A_{\mathrm{IP} 3}=10 \mathrm{~dB}\right)$.

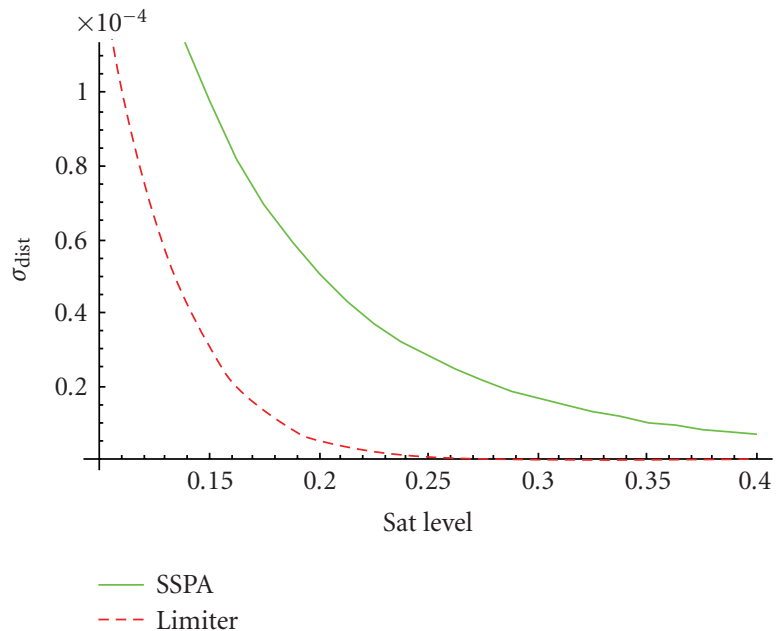

FIgURE 6: NLA distortion versus saturation level $a$, with $s=0.08$.

and finally

$$
\begin{aligned}
\sigma_{\mathrm{SSPA}}=\left(a e^{-a^{2} / 2 s^{2}} \sqrt{2 \pi}\left(a^{2}-s^{2}\right) \operatorname{erf} c\left[\frac{a}{\sqrt{2} s}\right]\right. \\
\left.+\frac{a^{4}}{2 s} e^{a^{2} / 2 s^{2}} E i\left[-\frac{a^{2}}{2 s^{2}}\right]-a^{2} s+2 s^{3}\right) .
\end{aligned}
$$

When plotting the deduced distortion models in (13), (17) versus the distribution parameter $(s)$ with saturation level $a=2\left(A_{\mathrm{IP} 3}=10 \mathrm{~dB}\right)$, we notice as shown in Figure 5 that the distortion due to the SSPA nonlinearity is much more larger than that of its limiting effect, also it is obvious that the distortion is highley senstive to any variation of the parameter $(s)$ as the slopes of the curves show.

On the other hand, when plotting distortion model versus the saturation level with parameter $(s=0.08)$ as depicted in Figure 6, it is shown that the distortion decays as the value of saturation level increases. 


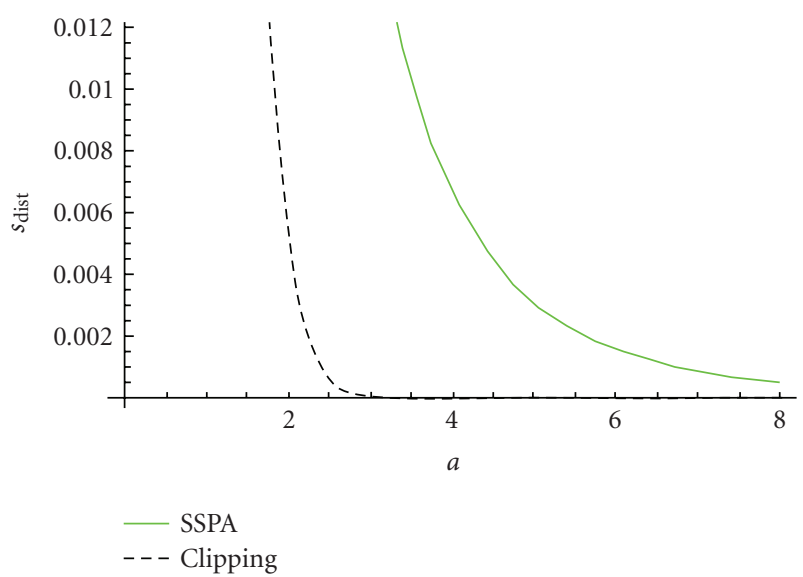

Figure 7: NLA distortion versus $a$, with $s=0.8$.

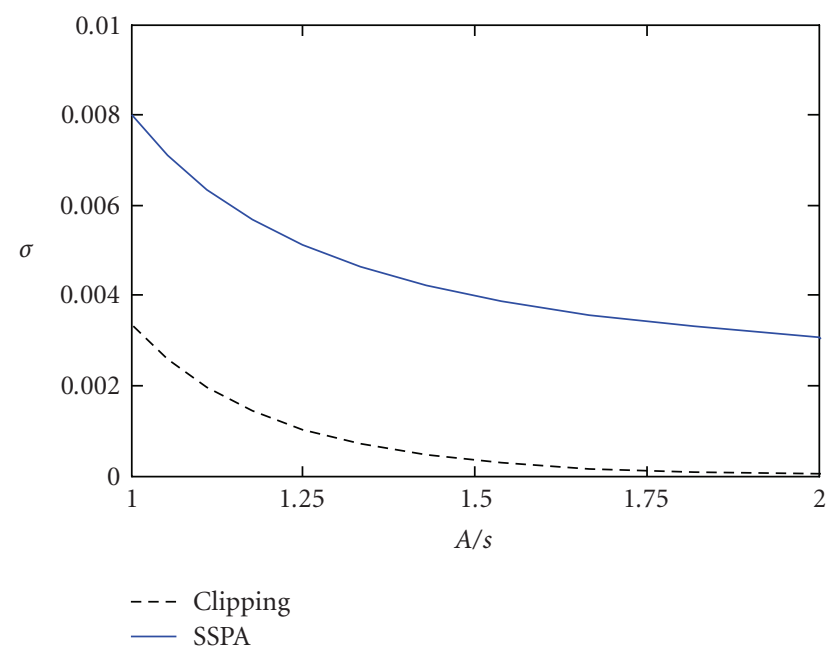

FIgURE 8: NLA distortion versus $A / s$.

And when plotting distortion model versus the saturation level with parameter $(s=0.8)$, Figure 7 shows a great increase in the distortion despite of the constant value of $(A / s)$.

And finally when plotting distortion model versus $(A / s)$ as shown in Figure 8 that shows that the distortion is reduced as the value of $(A / s)$ increases.

From Figures 5, 6, 7, and 8, it is clear that the distortion due to these effects is highly related to $(s)$ the distribution parameter, which controls the dynamic range itself, rather than the clipping level or the saturation level of the nonlinear amplifier.

\section{OFDM the Signal Quality Metrics}

\subsection{Error Vector Magnitude}

The modulation accuracy of the OFDM signal is measured by error vector magnitude. EVM is a measure for the difference between the theoretical wave and modified version of the

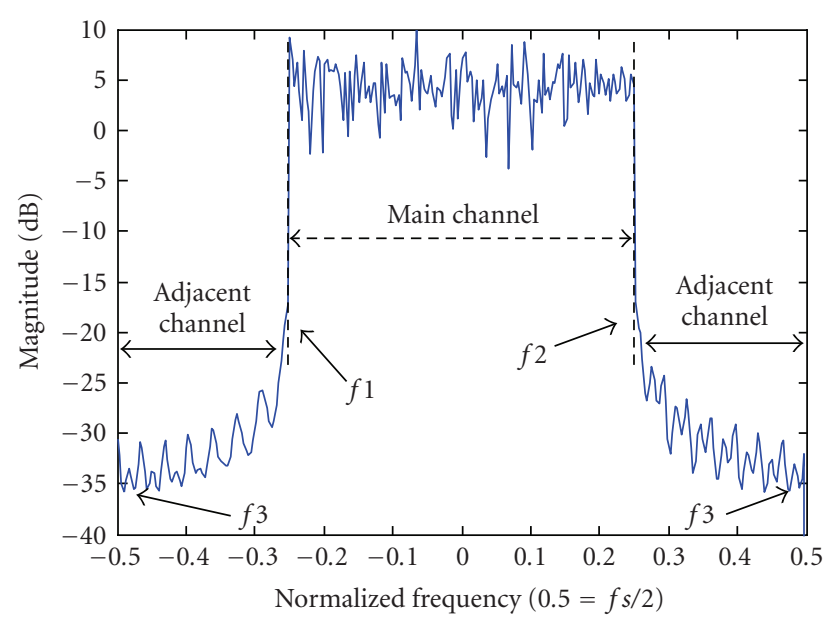

FIgURE 9: OFDM signal spectrum.

measured waveform. The measured waveform is modified by first passing it through a specified receiver measuring filter.

The waveform is further modified by selecting the frequency, absolute phase, absolute amplitude, and clock timing so as to minimize the error vector. The EVM result is defined as the square root of the ratio of the mean error vector power to the mean reference signal power expressed as a percentage. Mathematically, the error vector $e$ can be written as

$$
e=y-x
$$

where $y$ is the modified measured signal and $x$ the ideal transmitted signal. EVM can be defined as

$$
\mathrm{EVM}_{\mathrm{rms}}=\sqrt{\frac{E\left[|e|^{2}\right]}{E\left[|x|^{2}\right]}} .
$$

\subsection{Adjacent Channel Leakage Power Ratio (ACPR)}

Another figure of merit, specific to evaluate the out of band behavior of the HPA, is the ACPR; it should stay below the value specified. The ACPR is the ratio of the transmitted power to the power after a receiver filter in the adjacent channel.

In order to evaluate the ability of HPA models to reproduce the ACPR, we will use

$$
\triangle \mathrm{ACPR}=\operatorname{ACPR}(\tilde{S}(f))-\operatorname{ACPR}(S(f)),
$$

where $\widetilde{S}(f)$ is the PSD of the output of HPA while $S(f)$ is the true output.

The ACPR can be defined as

$$
\mathrm{ACPR}=\frac{\int_{f 2}^{f 3} S(f) d f}{\int_{f 1}^{f 2} S(f) d f}+\frac{\int_{f 1}^{f 4} S(f) d f}{\int_{f 1}^{f 2} S(f) d f},
$$

where $f_{1}$ and $f_{2}$ are the frequency limits of the main channel, and $f_{2}$ and $f_{3}$ are the limits of the upper adjacent channel, and $f_{1}$ and $f_{4}$ are the frequency limits of the lower adjacent channel as shown in Figure 9. 


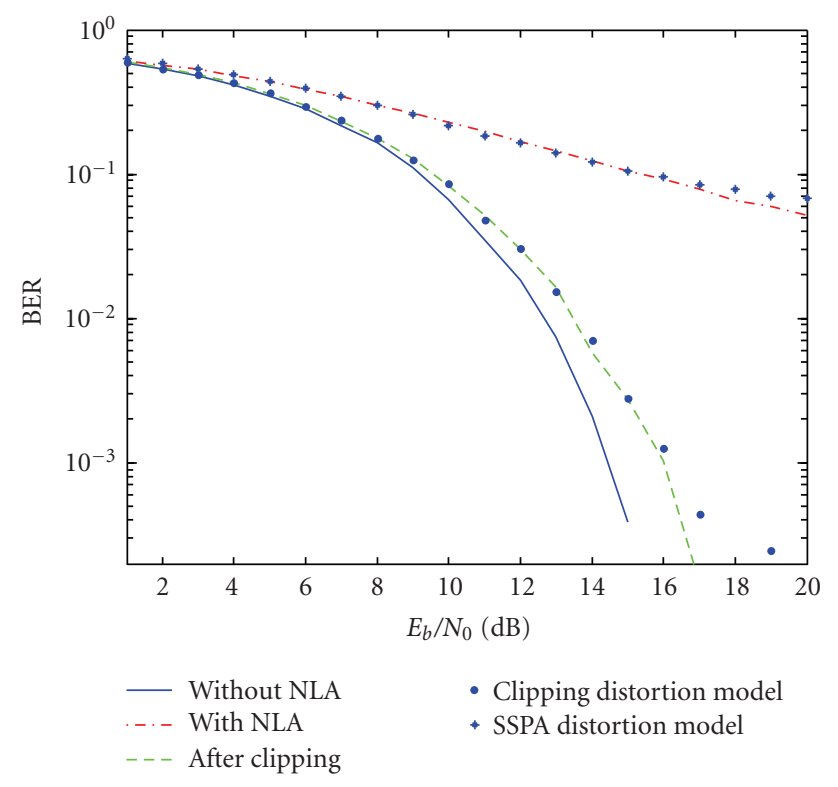

Figure 10: BER of OFDM system with HPA.

TABLE 1: ACPR and EVM for different limiting values.

\begin{tabular}{lcccc}
\hline \multirow{2}{*}{ Sat. level/ $Y_{\max }$} & \multicolumn{2}{c}{ ACPR $(\mathrm{dB})$} & \multicolumn{2}{c}{ EVM } \\
& Limiting & SSPA & Limiting & SSPA \\
\hline 1 & -44.7094 & -28.7268 & $1.3 e-016$ & 0.0929 \\
2 & -44.7094 & -31.0497 & $1.3 e-016$ & 0.0273 \\
10 & -44.7094 & -32.8791 & $1.3 e-016$ & 0.0012 \\
0.75 & -30.4841 & -27.2594 & 0.0126 & 0.1448 \\
0.5 & -23.4736 & -25.0624 & 0.0969 & 0.2474 \\
0.25 & -14.5232 & -22.3549 & 0.3972 & 0.4741 \\
\hline
\end{tabular}

\section{Simulation Results}

An OFDM system is implemented using 512 carriers with cyclic prefix length equal to 4. Each carrier is modulated using 16-QAM constellation. AWGN noise is included. BER simulations compared with theoretical results considering the power amplifier distortion models deduced above in (13), (17) are shown in Figure 10.

From this figure, it is possible to see that it was predicted in the previous analysis. The effect of nonlinear power amplifier is illustrated, where a limiter amplifier is included in the simulations with clipping levels of $12 \mathrm{~dB}$. The harmful effect of the nonlinearity can also be clearly seemed in this figure. Finally the figure shows that the computer simulations of BER are completely matched with the deduced models both the limiting and the nonlinearity effect.

Table 1 shows The ACPR value for both limiting and nonlinear effects with different limiting values relative to the maximum absolute value of the OFDM composite time signal $Y_{\max }$.

It is clear that as the limiting value decreases, the ACPR increases. It can also be noted that the effect of nonlinearity on ACPR value is negligible as compared to that of limiting as the clip level varies; this is due to the fact that the spectral

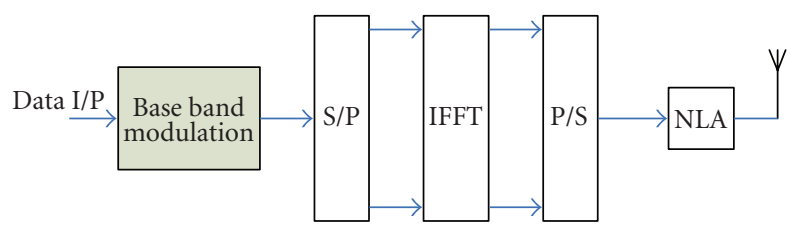

FIGURE 11: Block diagram of an OFDM system.

TABLE 2: PAR, STD, and dynamic range of OFDM signal.

\begin{tabular}{lccc}
\hline Modulation & PAR $(\mathrm{dB})$ & STD $(\delta)$ & Absolute signal range \\
\hline MSK & 8.3902 & 0.0427 & 0.0642 \\
QPSK & 8.7001 & 0.0562 & $0.0912 \equiv \mathrm{MSK}+3 \mathrm{~dB}$ \\
8-QAM & 9.0063 & 0.0965 & $0.1470 \equiv \mathrm{QPSK}+4 \mathrm{~dB}$ \\
16-QAM & 9.2989 & 0.1247 & $0.2213 \equiv 8 \mathrm{QAM}+3.5 \mathrm{~dB}$ \\
\hline
\end{tabular}

leakage that causes the ACPR to increase is mainly due to the clipping that can be viewed as windowing the spectrum by rectangular window. Table 1 shows also The EVM value for both limiting and nonlinear effects with different limiting values, it is clear that as the limiting value decreases, the EVM increases. And as the EVM is a measure of the total distortion, it is highly affected by the nonlinearity rather than the limiting effect.

\subsection{OFDM Using Different Modulators}

We have simulated an OFDM system shown in Figure 11 with 256 carriers and oversampling factor of 2 , with different modulation techniques, namely, $M$-QAM (with $M=4,8$, and 16) and MSK.

Table 2 Shows PAR, standard deviation $\delta$, and the dynamic range of the OFDM signal with the above mentioned modulation techniques. It is again in agreement with the above results. It is clear that although the PAR reduction due to the use of MSK instead of QPSK is slightly small, the true gain is the reduction in the dynamic range by $3 \mathrm{~dB}$, which enables us to use a low linearity and high-efficiency power amplifiers.

In addition, a new indicator arises in the table which is the standard deviation $\delta$, it is obvious from the table that MSK has the lowest $\delta$ while for $M$-QAM, as $M$ increase $\delta$ increases also, since we can deal with OFDM signal as a narrowband Gaussian noise with a mean of zero and variance of $\delta^{2}$, then $68 \%$ of amplitude values ranges in $[-\delta-\delta]$ and $99.994 \%$ of amplitude values ranges in $[-4 \delta-4 \delta]$, this can be a good indicator for clipping efficiency.

When applying SSPA amplifier using $v_{\text {sat }}=0.1$ on the OFDM signal, we have noticed the advantage of MSK over QPSK, as shown in Figure 12(a). Also, we have applied the amplifier on the OFDM signal using $M$-QAM with $M=4,8$, and 16. Using $v_{\text {sat }}=0.2$, we noticed that as $M$ increases, the distortion due to NLA increases and so the BER as shown in Figure 12(b).

As regarding to our previous results, it can be noticed that the MSK modulation gives us the lowest PAR when used in OFDM, besides its main advantage that it ignores 


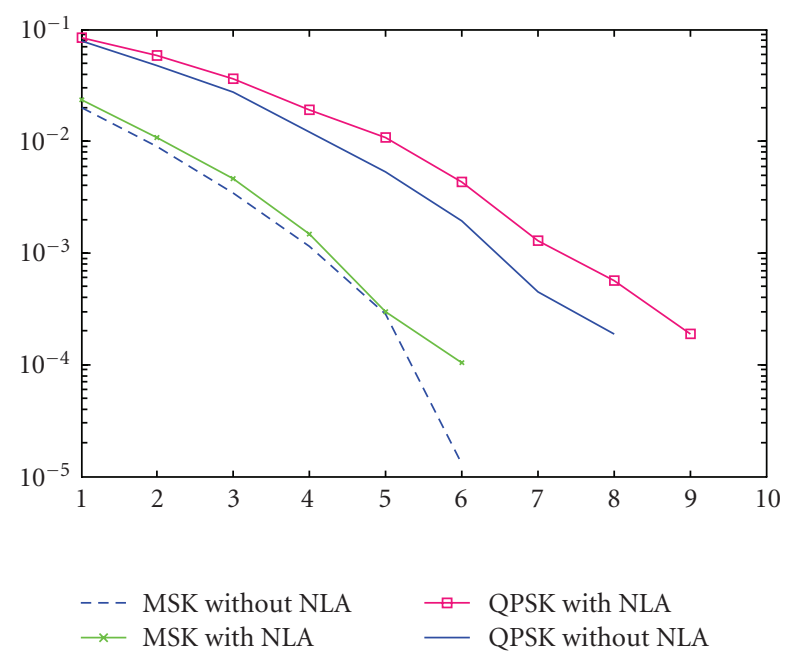

(a)

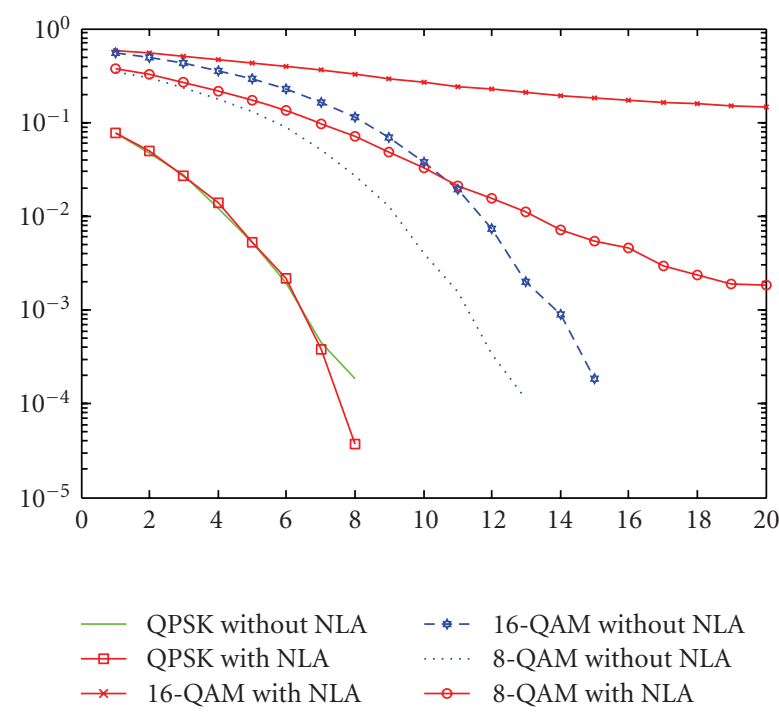

(b)

FIGURE 12: (a) Effect of NLA on MSK and QPSK. (b) Effect of NLA on $M$-QAM.

any fading-introduced amplitude fluctuation present in the received signal, and hence facilitates the utilization of power efficient class (C) amplifier [5].

\subsection{Walsh Hadamard Transform}

The Walsh-Hadamard transform (WHT) is perhaps the best known of the nonsinusoidal orthogonal transforms. The Walsh-Hadamard transform of a signal $x$, of size $N=2 n$, is the matrix-vector product $\mathrm{WHT}_{N} \cdot x,[6]$ where

$$
\mathrm{WHT}_{N}={\underset{\otimes}{i=1}}_{\otimes}^{n} \mathrm{DFT}_{2}=\overbrace{\mathrm{DFT}_{2} \otimes \cdots \otimes \mathrm{DFT}_{2}}^{n},
$$

$\mathrm{DFT}_{2}=(1 / 2)\left[\begin{array}{cc}1 & 1 \\ 1 & -1\end{array}\right]$ is the 2-point DFT matrix, and $\otimes$ denotes the tensor or Kronecker product. The tensor product of two matrices is obtained by replacing each entry of the

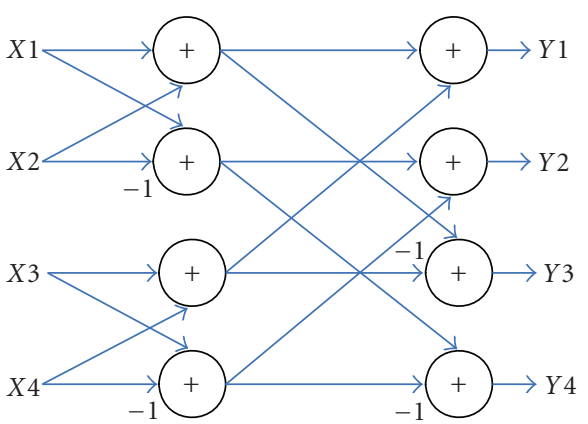

Figure 13: Four-point FWHT.

first matrix by that element multiplied by the second matrix. Thus, for example,

$$
\mathrm{WHT}_{4}=\frac{1}{2}\left[\begin{array}{cc}
1 & 1 \\
1 & -1
\end{array}\right] \otimes \frac{1}{2}\left[\begin{array}{cc}
1 & 1 \\
1 & -1
\end{array}\right]=\frac{1}{4}\left[\begin{array}{cccc}
1 & 1 & 1 & 1 \\
1 & -1 & 1 & -1 \\
1 & 1 & -1 & -1 \\
1 & -1 & -1 & 1
\end{array}\right] .
$$

The WHT has gained prominence in digital signal processing applications, since it can be computed using additions and subtractions only. Consequently, its hardware implementation is simpler.

\subsection{Fast Walsh-Hadamard Transform (FWHT)}

As the FFT is an algorithm to compute the DFT efficiently, similarly the FWHT is an algorithm to compute the WHT efficiently. The FWHT can be expressed as [6]

$$
W(u)=\frac{1}{N} \sum_{x=0}^{N-1} f(x) g(x, u)
$$

where $g(x, u)=\left[\prod_{i=0}^{n-1}(-1)^{b_{i}(x) b_{n-1-i}(u)}\right]$.

The FWHT can be derived using matrix factoring or matrix partitioning techniques. The signal flow graph for a 4 point FWHT is shown in Figure 13.

In a digital system, the $1 / 4$ multiplier can be simply implemented in two arithmetic shifts. The number of additions and subtractions needed to compute the four WHT coefficients is $4 \times \log _{2} 4=8$.

\subsection{OFDM System Using FWHT}

We have examined the use of FWHT with OFDM with different lengths.

The idea: since the peak value of the OFDM symbol results at the instant of coherent addition of subcarriers, it is possible to reduce this peak by modifying the subcarriers initial phases so as to avoid this condition. This can be done via successive phase shifts, we have tried when summing up a number of orthogonal sinusoidal waveforms with successive 


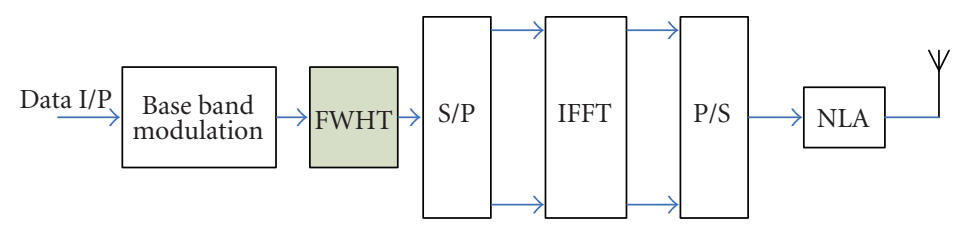

FIGURE 14: OFDM system block diagram.

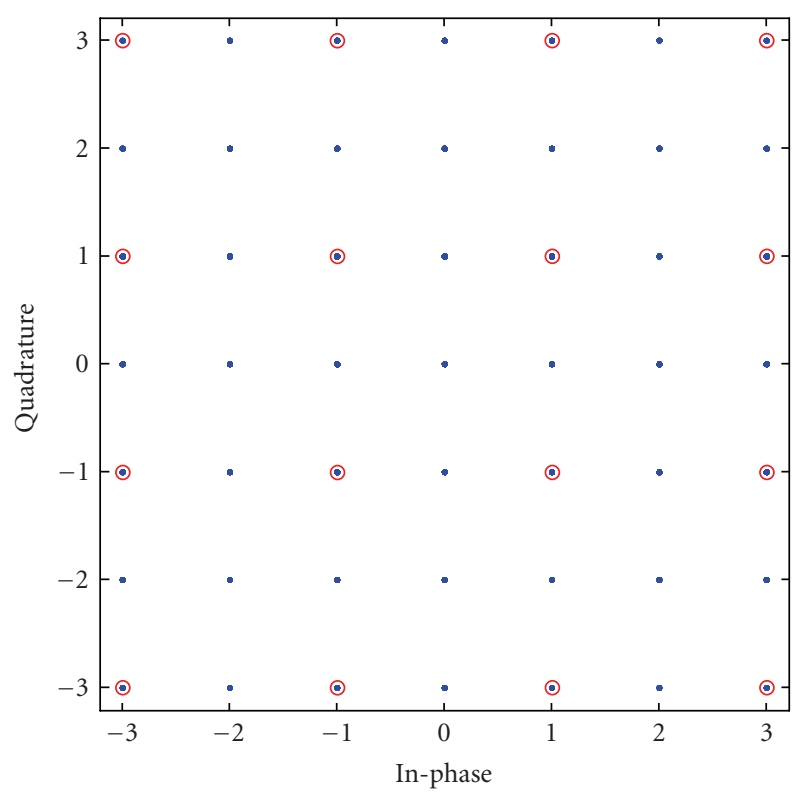

- 16-QAM with FWHT (2 points) O 16-QAM

Figure 15: Constellation diagram of OFDM signal.

phase shifts with different phase shift step in each time we try and we have got the optimum case at phase shift step $=\pi$ which is equivalent to modifying the OFDM symbol representation to be as follows:

$$
y_{n}=\sum_{k=0}^{N-1}(-1)^{k} X_{k} e^{j 2 \pi(n k / N)} .
$$

The above equation can be approximately implemented as shown in Figure 14 in the proposed block diagram of OFDM system.

In the simulations, 16 QAM modulation scheme is selected in OFDM with 512 subcarriers and an oversampling factor of 2, that is, IFFT length $=1024$.

Figure 15 depicts the constellation diagram of an OFDM mapped signal with and without FWHT, it is clear that the FWHT can be viewed as an active constellation extension (ACE) method, one of the latest PAR reduction, which alter or introduce new signal constellations to combat large signal peaks [7], but our concern here is its effect on the dynamic range of the OFDM signal. Simulations show that when using the OFDM shown in Figure 14 with 16-QAM without FWHT, the PAR is found to be $10.8 \mathrm{~dB}$, while when using the

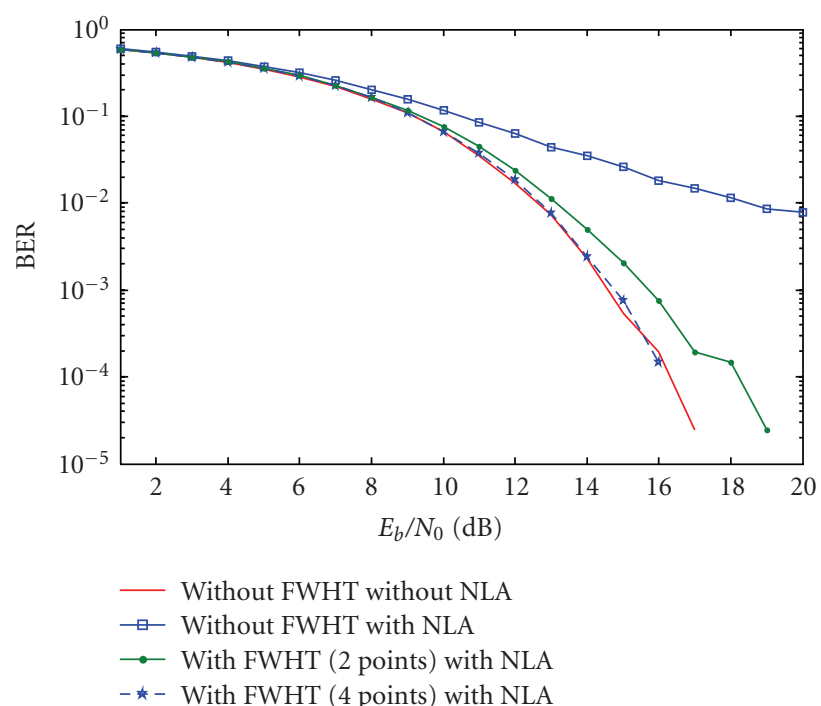

FIGURE 16: Effect of NLA on BER with/without FWHT.

FWHT ( 2 and 4 points), the PAR equals 10.5 and $10.15 \mathrm{~dB}$, respectively, provided that the dynamic range is reduced by 3 and $6 \mathrm{~dB}$ in the same cases, respectively.

The BER performance as a function of the signal-tonoise ratio (SNR) $E_{b} / N_{o}$ in OFDM systems with and without FWHT in the presence of NLA with $v_{\text {sat }}=0.1$ is depicted in Figure 16.

It can be noticed that the use of FWHT (4 points) greatly avoids the distortion due to NLA as compared to the 16 QAM case, despite the negligible reduction in the PAR. It is clear that the real reason in this good performance is the reduction in the dynamic range of about $6 \mathrm{~dB}$, again in a complete agreement with the above results.

\section{Conclusions}

In this paper, the effects of nonlinearities in the power amplifier over OFDM systems were analyzed and simulated. We can conclude that

(i) the distortion due to high-power amplifier, either limiting or nonlinearity effects, is highly related to the distribution parameter $(s)$ that controls the dynamic range itself rather than the clipping level or the saturation level of the amplifier;

(ii) also it is noticed that the effect of nonlinearity on ACPR value is negligible as compared to that of limiting as the clip level varies; this is due to the 
fact that the spectral leakage that causes the ACPR to increase is mainly due to the clipping that can be viewed as windowing the spectrum by rectangular window;

(iii) the EVM is a measure of the total distortion, it is highly affected by the nonlinearity rather than the limiting effect. And generally as the limiting value decreases, the EVM increases;

(iv) although the PAR reduction due to the use of MSK instead of QPSK is slightly small, the true gain is the reduction in the dynamic range by $3 \mathrm{~dB}$, which enables us to use a low linearity and high-efficiency power amplifiers like class (B) or (C);

(v) Walsh-Hadamard transform is used with OFDM systems as an intelligent scaling factor to reduce the dynamic range of the OFDM signal without the risk of amplifying the noise when restoring the signal to its original level. This technique offers an excellent solution to all of peak power problems in OFDM systems and without any loss in terms of spectral efficiency and without any side information being transmitted, and can be applied with low computational complexity;

(vi) the use of WHT with OFDM system enables the use of the high-efficiency class (C) amplifier without affecting the BER performance.

\section{References}

[1] F. H. Gregorio and T. I. Laakso, "The performance of OFDMSDMA systems with power amplifier non-linearities," in Proceedings of Finnish Signal Processing Symposium (FINSIG '05), pp. 58-61, Kuopio, Finland, August 2005.

[2] C. Schurgers, "Systematic approach to peak-to-average power ratio in OFDM," in Advanced Signal Processing Algorithms, Architectures, and Implementations XI, F. T. Luk, Ed., vol. 4474 of Proceedings of SPIE, pp. 454-464, San Diego, Calif, USA, August 2001.

[3] L. Liu, K. Hamaguchi, and H. Wakana, "Analysis of the combined effects of nonlinear distortion and phase noise on OFDM systems," IEICE Transactions on Communications, vol. E88-B, no. 1, pp. 304-310, 2005.

[4] S. V. Zhidkov, "Performance analysis of multicarrier systems in the presence of smooth nonlinearity," EURASIP Journal on Wireless Communications and Networking, vol. 2004, no. 2, pp. 335-343, 2004.

[5] T. Javornik, G. Kandus, and A. G. Burr, "The performance of N-GMSK signals in non-linear channels," in Proceedings of the 4th International Symposium on Wireless Personal Multimedia Communications (WMPC '01), Aalborg, Denmark, September 2001.

[6] M. Furis, P. Hitczenko, and J. R. Johnson, "Cache miss analysis of WHT algorithms," in Proceedings of International Conference on Analysis of Algorithms, Discrete Mathematics and Theoretical Computer Science (DMTCS '05), pp. 115-124, Nancy, France, October 2005.

[7] B. S. Krongold and D. L. Jones, "PAR reduction in OFDM via active constellation extension," IEEE Transactions on Broadcasting, vol. 49, no. 3, pp. 258-268, 2003. 

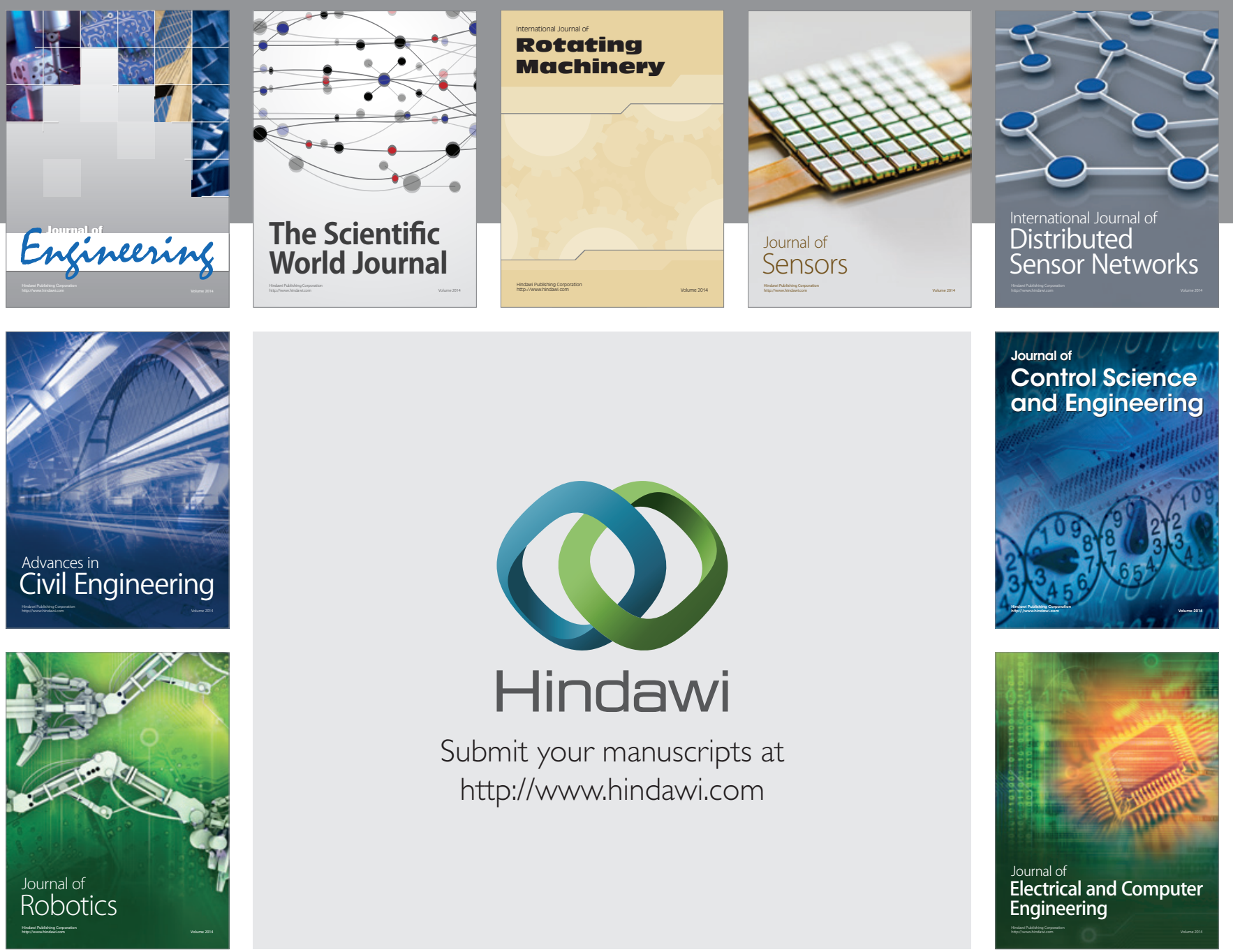

Submit your manuscripts at

http://www.hindawi.com
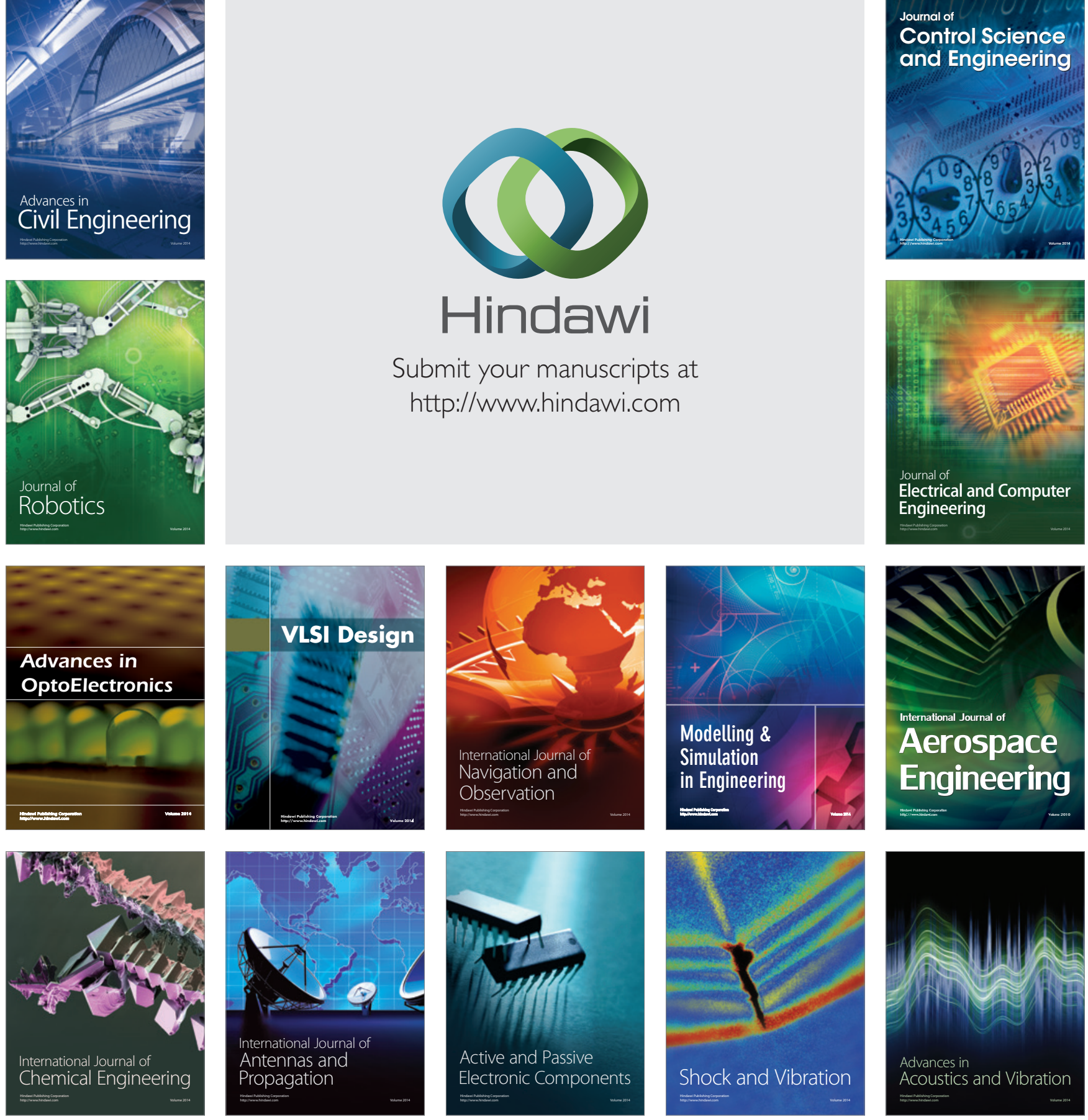